\title{
Patient-Reported Outcomes Measurement Information System Test Is Less Responsive Than Legacy Hip-Specific Patient-Reported Outcome Measures in Patients Undergoing Arthroscopy for Femoroacetabular Impingement Syndrome
}

\author{
Blake M. Bodendorfer, M.D., Ian M. Clapp, M.D., M.S., Robert B. Browning, M.D., \\ Thomas D. Alter, M.S., Benedict U. Nwachukwu, M.D., M.B.A., and \\ Shane J. Nho, M.D., M.S.
}

\begin{abstract}
Purpose: To evaluate and compare the Patient-Reported Outcomes Measurement Information System (PROMIS) Physical Function (PF) computerized adaptive test and PROMIS Pain Interference (PI) instruments versus legacy patientreported outcome measures (PROMs) in patients undergoing hip arthroscopy for femoroacetabular impingement syndrome at 1-year follow-up. Methods: Patients undergoing primary hip arthroscopy for the treatment of femoroacetabular impingement syndrome without concomitant procedures performed by a single surgeon between August 2018 and January 2019 were identified. The PROMIS PF score, PROMIS PI score, Hip Outcome Score-Activities of Daily Living Subscale (HOS-ADL), Hip Outcome Score-Sports Subscale (HOS-SS), modified Harris Hip Score (mHHS), International Hip Outcome Tool 12 (iHOT-12), and visual analog scale (VAS) pain score were obtained preoperatively and at 6 months and 1 year postoperatively. Bivariate correlation analyses between PROMIS and legacy PROMs were performed. The floor and ceiling effects, responsiveness, and relative efficiency (RE) of each PROM were calculated. Results: This study included 124 patients with an average age of $32.7 \pm 12.3$ years. The PROMIS PF score showed very good correlations with the HOS-ADL $(r=0.73, P<.001)$ and mHHS $(r=0.70, P<.001)$ and good correlations with the HOS-SS $(r=$ $0.62, P<.001)$, iHOT-12 score $(r=0.62, P<.001)$, and VAS pain score $(r=-0.64, P<.001)$. The PROMIS PI score showed very good inverse correlations with the HOS-ADL $(r=-0.72, P<.001)$ and mHHS $(r=-0.79, P<.001)$ and good correlations with the HOS-SS $(r=-0.64, P<.001)$, iHOT-12 score $(r=-0.65, P<.001)$, and VAS pain score $(r=$ $0.65, P<.001)$. No floor effect was observed for any measure. Ceiling effects were not observed in the PROMIS PROMs but were detected for the HOS-ADL (16.1\%) and mHHS (19.3\%). The effect size was large for all outcomes: iHOT-12 score, $d=1.77$; HOS-ADL, $d=1.37$; HOS-SS, $d=1.45$; PROMIS PI score, $d=1.05$; and PROMIS PF score, $d=1.01$. The iHOT-12 score was more responsive than the PROMIS PI score (RE $=1.69)$, PROMIS PF score $(\mathrm{RE}=1.75)$, HOS-ADL $(\mathrm{RE}=1.29)$, and HOS-SS (RE $=1.22)$. Conclusions: At 1-year follow-up, PROMIS PROMs displayed very good correlations with legacy PROMs. However, PROMIS PROMs had lower effect sizes and were not as responsive as legacy PROMs. Level of Evidence: Level II, development of diagnostic criteria on the basis of consecutive patients.
\end{abstract}

From the Department of Orthopaedic Surgery, Division of Sports Medicine, Rush University Medical Center, Chicago, Illinois, U.S.A. (B.M.B., I.M.C., R.B.B., T.D.A., S.J.N.); and Department of Orthopaedic Surgery, Hospital for Special Surgery, New York, New York, U.S.A. (B.U.N.).

The authors report the following potential conflicts of interest or sources of funding: B.U.N. receives intellectual property royalties from Remote Health, outside the submitted work. S.J.N. receives research support from Allosource, Arthrex, Athletico, DJ Orthopedics, Linvatec, Miomed, Smith $\theta$ Nephew, and Stryker; is a board or committee member of the American Orthopaedic Association, American Orthopaedic Society for Sports Medicine, and Arthroscopy Association of North America; receives intellectual property royalties from Ossur and Stryker; receives publishing royalties and financial/material support from Springer; and is a paid consultant for Stryker, outside the submitted work. Full ICMJE author disclosure forms are available for this article online, as supplementary material.

Received December 28, 2020; accepted July 24, 2021.

Address correspondence to Ian M. Clapp, M.D., M.S., Rush University Medical Center, 1611 W Harrison St, Chicago, IL 60612, U.S.A. E-mail: nho. research@rushortho.com

(C) 2021 THE AUTHORS. Published by Elsevier Inc. on behalf of the Arthroscopy Association of North America. This is an open access article under the CC BY-NC-ND license (http://creativecommons.org/licenses/by-nc-nd/4.0/). 2666-061X/202035

https://doi.org/10.1016/j.asmr.2021.07.020 
Ance its development in 2004 by the National $\checkmark$ Institutes of Health, the Patient-Reported Outcomes Measurement Information System (PROMIS) tool has been used increasingly in the orthopaedic literature. The PROMIS tool functions with integrated item response theory with computerized adaptive testing (CAT) to provide a single, generalizable and validated patient-reported outcome measure (PROM) while attempting to negate floor or ceiling effects with fewer individual questions when compared with legacy PROMs. ${ }^{1-5}$ A number of studies focusing on a variety of knee and shoulder injuries and surgical procedures have compared PROMIS measures with legacy PROMs in the past, showing good to excellent correlations with legacy measures. ${ }^{6-10}$ However, there is a relative paucity of literature comparing PROMIS measures with legacy PROMs in patients who have undergone hip arthroscopy for femoroacetabular impingement syndrome (FAIS), with past studies showing good to excellent correlations preoperatively and at 6-month follow-up but decreased responsiveness of the PROMIS Physical Function (PF) instrument at 6-month follow-up. ${ }^{11-15}$

Hip arthroscopy is an established procedure for FAIS with demonstrable improvement in PROMs such as the modified Harris Hip Score (mHHS), Hip Outcome Score (HOS), and International Hip Outcome Tool 12 (iHOT12) score. Although these hip-specific PROMs have been examined to understand their responsiveness and representative clinically significant changes, no studies have compared these legacy measures with PROMIS measures at 1 year postoperatively. Although the PROMIS tool does not have hip-specific questions, patients with hip and other lower-extremity conditions are defaulted to the PROMIS PF instrument. Little is known about whether this delineation is appropriate. The PROMIS PF CAT consists of an item bank of 165 questions, and patients complete between 4 and 12 questions, with the patients' responses guiding subsequent questions. The PROMIS Pain Interference (PI) instrument measures the consequences of pain and may include the extent to which pain hinders engagement with social, cognitive, emotional, physical, and recreational activities. ${ }^{16}$ Similarly to the PROMIS PF CAT, the PROMIS PI CAT consists of an item bank of 40 questions and patients complete between 4 and 12 questions. The PROMIS PI instrument also incorporates items regarding sleep and overall enjoyment in life. The PI form is universal rather than injury specific.

The purpose of the study was to evaluate and compare the PROMIS PF CAT and PROMIS PI instruments versus legacy PROMs in patients undergoing hip arthroscopy for FAIS at 1-year follow-up. We hypothesized that legacy and PROMIS PROMs would have good to excellent correlations and that legacy
PROMs would be more responsive with greater relative efficiencies (REs) when compared with PROMIS measures at 1-year follow-up for patients undergoing hip arthroscopy for the treatment of FAIS.

\section{Methods}

\section{Patient Selection}

After approval from our institutional review board, data were prospectively collected on all patients undergoing hip arthroscopy for the treatment of FAIS performed by a single, fellowship-trained and boardcertified orthopaedic surgeon (S.J.N.). Data in a clinical repository were retrospectively analyzed for patients undergoing primary hip arthroscopy for the treatment of FAIS between August 2018 and January 2019. The inclusion criteria consisted of a clinical and radiographic diagnosis of FAIS, failure of conservative management (including physical therapy, activity modification, oral anti-inflammatories, and/or intraarticular cortisone injections), and surgical treatment with hip arthroscopy for FAIS. ${ }^{17}$ Only patients completing at least 1 outcome measure preoperatively and at 1 year postoperatively were included. The exclusion criteria consisted of a history of bilateral hip surgery, hip arthroscopy for an indication other than FAIS, signs of osteoarthritis (Tönnis grade $>1$ ), hip dysplasia (lateral center-edge angle $<20^{\circ}$ ), a history of pediatric hip disorders (slipped capital femoral epiphysis, hip dysplasia, Perthes disease, and so on), concomitant trochanteric bursectomy, and concomitant gluteus medius or minimus repair.

\section{Functional Outcome Evaluation}

Preoperatively, demographic data were collected from all patients, including sex, age, operative extremity, body mass index, and comorbidities. Preoperatively and at 6 months and 1 year postoperatively, Patients completed legacy hip-specific PROMs including the HOS-Activities of Daily Living Subscale (HOS-ADL), ${ }^{18}$ HOS-Sports Subscale (HOS-SS), mHHS, ${ }^{19}$ and iHOT12 score. $^{20}$ Patients also completed the CAT version of the PROMIS PF and PROMIS PI instruments ${ }^{21}$ via the Outcome Based Electronic Research Database (OBERD; Universal Research Solutions, Columbia, MO).

\section{Statistical Analysis}

Analysis was performed to identify any existing floor and ceiling effects for the legacy hip-specific PROMs, PROMIS PF measure, and PROMIS PI measure. For the legacy PROMs, any percentage of patients greater than or equal to $15 \%$ of the study population achieving the maximum or minimum score was deemed a significant ceiling or floor effect. ${ }^{22}$ For the PROMIS measures, any percentage greater than or equal to $15 \%$ of the study 
Table 1. Floor and Ceiling Effects of Postoperative Outcome Scores

\begin{tabular}{lcc}
\hline & Floor, $\%$ & Ceiling, $\%$ \\
\hline HOS-ADL & 0 & $16.1^{*}$ \\
HOS-SS & 0 & 12.1 \\
iHOT-12 score & 0 & 3.3 \\
mHHS & 0 & $19.3^{*}$ \\
PROMIS Pain Interference score & 3.3 & 7.4 \\
PROMIS Physical Function score & 4.8 & 3.2
\end{tabular}

HOS-ADL, Hip Outcome Score-Activities of Daily Living Subscale; HOS-SS, Hip Outcome Score-Sports Subscale; iHOT-12, International Hip Outcome Tool 12; mHHS, modified Harris Hip Score; PROMIS, Patient Reported Outcomes Measurement Information System.

*For ceiling or floor effects, at least $15 \%$ of patient outcomes had to achieve the maximum or minimum score.

population in the top or bottom fifth percentile was deemed a significant ceiling or floor effect. ${ }^{7}$ Pearson coefficient analysis was used to identify correlations between PROMIS scores and the legacy PROMs. Correlation was defined as follows: excellent, greater than 0.80 ; very good, 0.71 to 0.80 ; good, 0.61 to 0.70 ; fair, 0.41 to 0.60 ; or poor, 0.21 to $0.40 .^{14}$

To directly compare the responsiveness between PROMs, the effect size and RE were calculated for each PROM tool. ${ }^{23,24}$ The effect size, or Cohen $d$, is a measure of the magnitude of the preoperative-topostoperative change in relation to the amount of variability in the scores. The Cohen $d$ was calculated by dividing the absolute difference in the mean change score for each PROM by the pooled standard deviation for that PROM tool. The RE was calculated to directly compare responsiveness between PROM tools. ${ }^{23,25}$ The $\mathrm{RE}$ is the $t$ score-the ratio between the difference between the 2 groups and the difference within the groups derived from the paired $t$ test of the respective preoperative and 1-year PROM scores-from 1
PROM tool divided by the $t$ score of another PROM tool and then squared. If the RE was greater than 1 , the first PROM would be considered "more responsive" than the second PROM tool; if it was less than l, the first PROM tool would be considered "less responsive" than the second PROM tool. These measures allow one to compare the psychometric properties of the PROMs and determine whether they are suitable for detecting changes in function in the postoperative period.

Descriptive statistics for all continuous variables are reported as means with standard deviations, and frequency statistics are reported for all non-continuous variables. Paired-samples $t$ tests were used to compare preoperative and 1-year postoperative PROMs. Statistical significance for all analyses was set at $\alpha \leq .05$. All statistical analyses were performed using SPSS software (version 26; IBM, Armonk, NY).

\section{Results}

\section{Demographic Characteristics}

A total of 222 patients underwent primary hip arthroscopy between August 2018 and January 2019. Of these patients, 9 underwent hip arthroscopy for an indication other than FAIS, 18 underwent prior ipsilateral or contralateral hip arthroscopy, 3 had a history of pediatric hip disorders or dysplasia, and 38 were missing preoperative PROMIS PROMs and were excluded. A total of 124 patients completed at least 1 preoperative PROM and at least 1 PROM at a minimum of 1-year follow-up and were included in the analysis; the average follow-up period was $13.7 \pm 2.1$ months. Female patients $(73.4 \%)$ comprised the majority of this cohort; the average age and body mass index were $32.7 \pm 12.3$ years and $26.2 \pm 6.9 \mathrm{~kg} / \mathrm{m}^{2}$, respectively.

Table 2. Correlation Analysis of Preoperative and 1-Year Postoperative Patient-Reported Outcomes

\begin{tabular}{|c|c|c|c|c|}
\hline \multicolumn{5}{|l|}{ Preoperative } \\
\hline HOS-SS & 0.616 & $<.0001^{*}$ & -0.574 & $<.0001^{*}$ \\
\hline iHOT-12 score & 0.710 & $<.0001^{*}$ & -0.647 & $<.0001^{*}$ \\
\hline mHHS & 0.675 & $<.0001^{*}$ & -0.690 & $<.0001^{*}$ \\
\hline HOS-ADL & 0.729 & $<.0001^{*}$ & -0.721 & $<.0001^{*}$ \\
\hline HOS-SS & 0.621 & $<.0001^{*}$ & -0.635 & $<.0001^{*}$ \\
\hline iHOT-12 score & 0.622 & $<.0001^{*}$ & -0.654 & $<.0001^{*}$ \\
\hline mHHS & 0.701 & $<.0001^{*}$ & -0.788 & $<.0001^{*}$ \\
\hline VAS pain score & -0.642 & $<.0001^{*}$ & 0.658 & $<.0001^{*}$ \\
\hline
\end{tabular}

HOS-ADL, Hip Outcome Score-Activities of Daily Living Subscale; HOS-SS, Hip Outcome Score-Sports Subscale; iHOT-12, International Hip Outcome Tool 12; mHHS, modified Harris Hip Score; PROMIS, Patient Reported Outcomes Measurement Information System; VAS, visual analog scale.

*Statistically significant. 
Table 3. Preoperative Versus Postoperative Outcome Score Difference and Pooled Effect Size

\begin{tabular}{lccc}
\hline & Mean Score Difference & Pooled SD & Pooled Effect Size $(d)$ \\
\hline PROMIS Pain Interference score & -11.04 & 10.47 & 1.05 \\
PROMIS Physical Function score & 10.78 & 10.62 & 1.01 \\
HOS-ADL & 26.03 & 19.00 & 1.37 \\
HOS-SS & 39.57 & 27.27 & 1.45 \\
mHHS & 23.63 & 17.92 & 1.32 \\
iHOT-12 score & 43.54 & 24.61 & 1.77 \\
\hline
\end{tabular}

HOS-ADL, Hip Outcome Score-Activities of Daily Living Subscale; HOS-SS, Hip Outcome Score-Sports Subscale; iHOT-12, International Hip Outcome Tool 12; mHHS, modified Harris Hip Score; PROMIS, Patient Reported Outcomes Measurement Information System; SD, standard deviation.

\section{Clinical Outcomes Analysis}

There were statistically significant improvements in all PROMs at 1-year follow-up: PROMIS PF (40.4 \pm 6.5 vs $51.2 \pm 10.9, P<.001)$, HOS-ADL $(62.4 \pm 17.0$ vs 88.4 $\pm 13.1, P<.0001)$, HOS-SS $(40.0 \pm 20.7$ vs $79.6 \pm 22.9, P$ $<.0001)$, mHHS $(60.2 \pm 15.0$ vs $85.0 \pm 15.8, P<.0001)$, and iHOT-12 score $(31.9 \pm 18.0$ vs $62.3 \pm 33.5, P<$ $.0001)$. The PROMIS PI score $(61.6 \pm 6.2$ vs $50.6 \pm 9.2, P$ $<.001)$ and VAS pain score $(55.0 \pm 18.7$ vs $17.8 \pm 19.6, P$ $<.001)$ showed statistically significant improvements at 1 year after surgery.

Floor and ceiling effects at 1 year postoperatively for all PROMs are summarized in Table 1. Ceiling effects were observed for the HOS-ADL and mHHS, with $16.1 \%$ and $19.3 \%$ of patients, respectively, achieving the maximum scores. No floor effect was observed for any measure.

\section{Correlation Analysis}

The results of Pearson coefficient analysis between PROMIS outcome and legacy PROM scores are summarized in Table 2. Preoperatively, the PROMIS PF score showed a very good correlation with the iHOT-12 score and good correlations with the HOS-ADL, HOSSS, and mHHS. The PROMIS PI score showed a fair correlation with the HOS-SS and good correlations with the HOS-ADL, iHOT-12 score, and mHHS. At 1 year postoperatively, the PROMIS PF score showed a very good correlation with the HOS-ADL and good correlations with the HOS-SS, mHHS, and HHOT-12 score. The
PROMIS PI score showed very good correlations with the HOS-ADL and mHHS and good correlations with the HOS-SS and iHOT-12 score.

\section{Responsiveness Analysis}

The pooled effect sizes for each PROM can be found in Table 3. The PROMIS outcomes had smaller mean differences and smaller pooled standard deviations compared with the legacy PROMs. The pooled effect size was large for all PROMs.

The REs between outcomes are summarized in Table 4. A value greater than 1 indicates that the PROM tool in the left column is more responsive than the PROM tool in the top row. The iHOT-12 score was the most responsive PROM, being more responsive than the PROMIS PI score, PROMIS PF score, HOS-ADL, and HOS-SS. The PROMIS PF score was overall the least responsive across all PROMs.

\section{Discussion}

In this study, we found that both the PROMIS PF and PROMIS PI instruments show good to very good correlations with legacy hip-specific PROMs. We also found that at minimum 1-year follow-up, there were no floor effects for any PROM; however, there were ceiling effects for the HOS-ADL and mHHS, with $16.1 \%$ and $19.3 \%$ of patients, respectively, achieving the highest possible scores. When comparing responsiveness, we found that the effect size was large for all outcomes (Cohen $d>1$ ) but was largest for the iHOT-12 score. The PROMIS PF

Table 4. Relative Efficiency Between Preoperative and 1-Year Postoperative Outcome Measures

\begin{tabular}{|c|c|c|c|c|c|}
\hline & $\begin{array}{l}\text { PROMIS Pain } \\
\text { Interference Score }\end{array}$ & $\begin{array}{l}\text { PROMIS Physical } \\
\text { Function Score }\end{array}$ & HOS-ADL & HOS-SS & iHOT-12 Score \\
\hline PROMIS Pain Interference score & - & 1.04 & 0.77 & 0.72 & 0.59 \\
\hline PROMIS Physical Function score & 0.96 & - & 0.74 & 0.70 & 0.57 \\
\hline HOS-SS & 1.38 & 1.44 & 1.06 & - & 0.82 \\
\hline mHHS & 1.08 & 1.12 & 0.93 & 0.74 & 0.80 \\
\hline iHOT-12 score & 1.69 & 1.75 & 1.29 & 1.22 & - \\
\hline
\end{tabular}

NOTE. A value greater than 1 indicates that the patient-reported outcome measure in the left column is more responsive than the patientreported outcome measure in the top row.

HOS-ADL, Hip Outcome Score-Activities of Daily Living Subscale; HOS-SS, Hip Outcome Score-Sports Subscale; iHOT-12, International Hip Outcome Tool 12; mHHS, modified Harris Hip Score; PROMIS, Patient Reported Outcomes Measurement Information System. 
score was overall the least responsive (with the PROMIS PI score being marginally more responsive), and the iHOT-12 score was more responsive than the HOS-SS, HOS-ADL, PROMIS PI score, and PROMIS PF score. These findings confirmed our hypothesis.

There is a relative paucity of literature regarding the psychometric properties of PROMIS scores in patients after hip arthroscopy for FAIS. In 2017, Sheean et al. ${ }^{26}$ first showed that PROMIS scores were significantly different between FAIS patients and controls, establishing the PROMIS instrument as a measure to potentially capture the disability associated with the syndrome. Nwachukwu et al. ${ }^{14}$ evaluated the preoperative performance of the PROMIS PF score in 197 nonoperative patients with FAIS. They found good to excellent Pearson correlations between the PROMIS score and the iHOT-12 score, HOS-ADL, HOS-SS, and mHHS. Each PROM was measured preoperatively in a cohort in which $100 \%$ of patients received a diagnosis of FAIS and were indicated for hip arthroscopic surgery. The study was limited, however, by a lack of postoperative follow-up and analysis. After this work, Nwachukwu et al. ${ }^{15}$ evaluated the performance of the PROMIS PF and PROMIS PI scores in patients with a minimum 6-month follow-up after hip arthroscopy for FAIS. In contrast to the high performance of the PROMIS PF score preoperatively, the authors found that the PROMIS PF score had a lower effect size compared with legacy PROMs and that there was a worse correlation with the iHOT-12 score as compared with the HOS-SS. Overall, the iHOT-12 score was the most responsive and had the largest effect size. Given that there is continued improvement in function in the postoperative period and PROMIS measures have been shown to be less responsive than legacy PROMs, it is necessary to compare how well PROMIS measurements are able to detect this improvement at multiple time points in the postoperative period. When compared with our study, the correlations at 6-month follow-up between the PROMIS PROMs and legacy PROMs were slightly stronger.

In this study, we found that the PROMIS PF and PROMIS PI measures correlated well with legacy hipspecific PROMs, with the best correlation shown for the HOS-ADL and mHHS. However, it should be noted that none of these correlations were excellent. At 1 year postoperatively, the best correlation between the PROMIS CAT and legacy PROMs was with the HOS-ADL and mHHS and the worst was with the HOS-SS and iHOT-12 score. This finding is important because both the HOS-SS and iHOT-12 score showed the highest responsiveness and the iHOT-12 score showed the highest RE over other PROMs. In this context, it should also be considered that neither the HOS-SS nor the iHOT-12 score had substantial ceiling effects. Simultaneously, the PROMIS PF and PROMIS PI scores showed the lowest responsiveness and lowest RE, which indicates that these measures may not be suitable to fully detect changes in function at 1-year follow-up in patients undergoing hip arthroscopy for FAIS. These data underscore the caution that we should take prior to replacing legacy PROMs with the PROMIS CAT. Given these findings, the PROMIS CAT may not completely gather all dimensions of hip-specific disability in FAIS compared with other hip-specific legacy PROMs.

\section{Limitations}

This study is not without limitations. The major limitation in this study is the limited follow-up period of 1 year postoperatively. Although we can support the use of the PROMIS tool and how it correlates with legacy PROMs over a l-year period, we are unable to predict further outcomes. Additionally, our study population consisted of patients who underwent hip arthroscopy with routine capsular closure, and thus, we are unable to evaluate the correlation between PROMIS measures and legacy PROMs for patients not undergoing surgical intervention. Next, this is a single-surgeon series, with all surgical procedures being performed by a highvolume hip arthroscopist, and therefore, there may be limited generalizability. Finally, this study may be limited by its sample size, as well as its nonrandomized retrospective study design.

\section{Conclusions}

At 1-year follow-up, PROMIS PROMs displayed very good correlations with legacy PROMs. However, PROMIS PROMs had lower effect sizes and were not as responsive as legacy PROMs.

\section{References}

1. Cella D, Gershon R, Lai JS, Choi S. The future of outcomes measurement: Item banking, tailored short-forms, and computerized adaptive assessment. Qual Life Res 2007;16: 133-141 (suppl 1).

2. Fries JF, Witter J, Rose M, Cella D, Khanna D, MorganDeWitt E. Item response theory, computerized adaptive testing, and PROMIS: Assessment of physical function. J Rheumatol 2014;41:153-158.

3. Chang $\mathrm{CH}$, Reeve $\mathrm{BB}$. Item response theory and its applications to patient-reported outcomes measurement. Eval Health Prof 2005;28:264-282.

4. Engelhard MM, Schmidt KM, Engel CE, Brenton JN, Patek SD, Goldman MD. The e-MSWS-12: Improving the multiple sclerosis walking scale using item response theory. Qual Life Res 2016;25:3221-3230.

5. Wylie JD, Beckmann JT, Granger E, Tashjian RZ. Functional outcomes assessment in shoulder surgery. World $J$ Orthop 2014;5:623-633.

6. Anthony CA, Glass N, Hancock K, Bollier M, Hettrich CM, Wolf BR. Preoperative performance of the PatientReported Outcomes Measurement Information System in patients with rotator cuff pathology. Arthroscopy 2017;33:1770-1774.el. 
7. Anthony CA, Glass NA, Hancock K, Bollier M, Wolf BR, Hettrich CM. Performance of PROMIS instruments in patients with shoulder instability. Am J Sports Med 2017:45:449-453.

8. Dowdle SB, Glass N, Anthony CA, Hettrich CM. Use of PROMIS for patients undergoing primary total shoulder arthroplasty. Orthop J Sports Med 2017;5:23259671 17726044.

9. Hancock KJ, Glass N, Anthony CA, et al. Performance of PROMIS for healthy patients undergoing meniscal surgery. J Bone Joint Surg Am 2017;99:954-958.

10. Hancock KJ, Glass N, Anthony CA, et al. PROMIS: A valid and efficient outcomes instrument for patients with ACL tears. Knee Surg Sports Traumatol Arthrosc 2019;27:100-104.

11. Nwachukwu BU, Chang B, Adjei J, et al. Time required to achieve minimal clinically important difference and substantial clinical benefit after arthroscopic treatment of femoroacetabular impingement. Am J Sports Med 2018;46: 2601-2606.

12. Nwachukwu BU, Chang B, Fields K, et al. Defining the "substantial clinical benefit" after arthroscopic treatment of femoroacetabular impingement. Am J Sports Med 2017:45:1297-1303.

13. Nwachukwu BU, Fields K, Chang B, Nawabi DH, Kelly BT, Ranawat AS. Preoperative outcome scores are predictive of achieving the minimal clinically important difference after arthroscopic treatment of femoroacetabular impingement. Am J Sports Med 2017;45:612-619.

14. Nwachukwu BU, Beck EC, Chapman R, Chahla J, Okoroha K, Nho SJ. Preoperative performance of the PROMIS in patients undergoing hip arthroscopic surgery for femoroacetabular impingement syndrome. Orthop $J$ Sports Med 2019;7:2325967119860079.

15. Nwachukwu BU, Rasio J, Beck EC, et al. PROMIS Physical Function has a lower effect size and is less responsive than legacy hip specific patient reported outcomes measures following arthroscopic hip surgery. Arthroscopy 2020;36: 2992-2997.

16. Amtmann D, Cook KF, Jensen MP, et al. Development of a PROMIS item bank to measure pain interference. Pain 2010;150:173-182.

17. Griffin DR, Dickenson EJ, O'Donnell J, et al. The Warwick Agreement on femoroacetabular impingement syndrome
(FAI syndrome): An international consensus statement. Br J Sports Med 2016;50:1169-1176.

18. Martin RL, Kelly BT, Philippon MJ. Evidence of validity for the Hip Outcome Score. Arthroscopy 2006;22: 1304-1311.

19. Chahal J, Van Thiel GS, Mather RC III, et al. The patient acceptable symptomatic state for the modified Harris Hip Score and Hip Outcome Score among patients undergoing surgical treatment for femoroacetabular impingement. Am J Sports Med 2015;43:1844-1849.

20. Nwachukwu BU, Chang B, Beck EC, et al. How should we define clinically significant outcome improvement on the iHOT-12? HSS J 2019;15:103-108.

21. Kollmorgen RC, Hutyra CA, Green C, Lewis B, Olson SA, Mather RC III. Relationship between PROMIS computer adaptive tests and legacy hip measures among patients presenting to a tertiary care hip preservation center. Am J Sports Med 2019;47:876-884.

22. Terwee CB, Bot SD, de Boer MR, et al. Quality criteria were proposed for measurement properties of health status questionnaires. J Clin Epidemiol 2007;60:34-42.

23. Kenney RJ, Houck J, Giordano BD, Baumhauer JF, Herbert M, Maloney MD. Do Patient Reported Outcome Measurement Information System (PROMIS) scales demonstrate responsiveness as well as disease-specific scales in patients undergoing knee arthroscopy? Am J Sports Med 2019;47:1396-1403.

24. Khalil LS, Darrith B, Franovic S, Davis JJ, Weir RM, Banka TR. Patient-Reported Outcomes Measurement Information System (PROMIS) global health short forms demonstrate responsiveness in patients undergoing knee arthroplasty. J Arthroplasty 2020;35:1540-1544.

25. Unger RZ, Burnham JM, Gammon L, Malempati CS, Jacobs CA, Makhni EC. The responsiveness of patientreported outcome tools in shoulder surgery is dependent on the underlying pathological condition. Am J Sports Med 2019;47:241-247.

26. Sheean AJ, Schmitz MR, Ward CL, et al. Assessment of disability related to femoroacetabular impingement syndrome by use of the Patient-Reported Outcome Measure Information System (PROMIS) and objective measures of physical performance. Am J Sports Med 2017;45: 2476-2482. 